\title{
Performance in den byzantinischen Romanen des 12. Jahrhunderts und das Theatron
}

\author{
Katalin L. Delbó
}

\section{Performance in the Byzantine Novels and the Theatron}

\begin{abstract}
During the 12th century, the history of the European novel came to a turning point. While the so-called chanson de geste and the early Arthurian novels gained prominence in Western Europe, the Byzantine Empire could welcome four new love novels after an eight-century break. One of these novels is Drosilla and Charicles by Nicetas Eugenianos. The Comnenian novels might have been written for aristocrat patrons and the Byzantine intelligentsia. We may also presume that these books were recited, either in smaller sections or in their full form, to an audience in the salons of the age, namely the theatra. Very few investigations have been made so far into the question of how we could find proof for the recitals. This article scrutinizes the signs that may prove these novels to have been performed, based on Nicetas Eugenianos. The study offers two perspectives on this topic: on the one hand, rhetorics and narratology areuge involved in the scrutiny (closely in line with our knowledge on theatron); on the other hand, performative scenes in the plot of the novel are also analyzed (e.g. the dance of the old lady Baryllis). These investigations confirm the fact that Drosilla and Charicles is a significant point in the development of the novel.
\end{abstract}

\section{Key words}

performance; theatron; byzantine novels; Nicetas Eugenianos; Drosilla and Charicles 
Es gibt nichts Neues unter der Sonne - sagt der biblische Spruch. Bis zur Mitte des 20. Jahrhunderts betonte die gelehrte Welt dasselbe im Zusammenhang mit der byzantinischen Literatur, insbesondere mit den vier Liebesromanen aus dem 12. Jahrhundert. In der Fachliteratur ist es oft zu lesen, dass diese Werke - nämlich Rhodanthe und Dosikles von Theodoros Prodromos, Hysmine und Hysminias von Eusthatios Makrembolites, Drosilla und Charikles von Niketas Eugenianos und Aristandros und Kallithea von Konstantinos Manasses - nur einfache, schwache Kopien der antiken Vorbilder sind. In den letzten Jahren beschäftigten und beschäftigen sich immer mehr Forscher mit den Neuerungen und der Charakteristik der einzelnen Romane und verwenden literaturwissenschaftliche Methoden, Theorien in ihren Untersuchungen. ${ }^{1}$ Durch ihre Arbeit ist das falsche Bild von diesen vier Romanen der Komnenenzeit heutzutage schon mehr oder weniger in den Hintergrund gedrängt worden, aber in der Forschung ergeben sich immer noch zahlreiche offene Fragen. Eine von ihnen ist die gegenseitige Beziehung der Gattung des Romans und der Oralität. Was weist darauf hin, oder woraus lässt sich im Text darauf schließen, dass diese Werke vor dem Publikum, möglicherweise im sogenannten theatron vorgelesen wurden? Mit dieser Frage steht eigentlich eine andere interessante Problematik eng im Zusammenhang: die Untersuchung des Performance-Motivs in den byzantinischen Romanen. Welche Rolle spielt dieses Motiv in den Werken und wie lässt es sich mit theatron vergleichen?

In der Romanforschung ist dieses Thema nicht vollständig neu. Margaret Mullet warf es schon in Verbindung mit Hysmine und Hysminias von Makrembolites in den achtziger Jahren auf und vor wenigen Jahren stellte Elisabeth Jeffreys diese Frage im Bezug allgemein auf byzantinische Romane. ${ }^{2}$ Ähnlichen Weg schlägt auch Panagiotis Agapitos ein, der - als erster - in seinem Beitrag mit dem Titel Writing, Reading and Reciting (in) Byzantine Erotic Fiction die drei zur Gänze überlieferten Romane analysierte und sehr wichtige Feststellungen traf. ${ }^{3}$ Es fehlt jedoch immer noch eine solche Arbeit, die die „Performance-Zeichen“ und das „Theatron-Motiv“ in einem dieser Liebesromane gründlich untersucht. Genau das ist das Vorhaben dieser Studie: ein erster Versuch der oben Erwähnten - hauptsächlich anhand des Romans von Niketas Eugenianos.

Vor der Analyse des Werkes von Eugenianos werfen wir einen kurzen Blick auf das literarische und kulturelle Milieu, in dem die byzantinischen Romanen erschienen. Das 12. Jahrhundert war in Byzanz eine aetas rhetorica: ${ }^{4}$ die Rhetorik bildete den Mittelpunkt

1 Einige wichtige Werke ohne Anspruch auf Vollständigkeit: Hunger (1980); Beaton (1996); MacAlister (1996); Agapitos \& Reinsch (2000); Nilsson (2001); Roilos (2005); Nilsson (2014).

2 "Again ambiguous, but possibly indicative is the description by Eusthatios Makrembolites of his novel Hysmine and Hysminias as a drama: where should a drama be performed but in a theatron?" Mullett berief sich auf M. Alexiou und H. Hunger (Mullett, 1984: pp. 175, 190); „R\&D, like other three novels from this period presented here, survives in written form but almost certainly would initially have been presented in a performance context, in a theatron either book by book or perhaps as a selection of detachable highlights" (Jeffreys, 2014: p. 14).

3 Agapitos (2006).

4 Roilos (2005: p. 27), im Allgemeinem zur Rhetorik im Byzanz des 12. Jahrhunderts siehe: Roilos (2005: pp. 27-32), über Mimesis und Rhetorik: Beaton (1996: pp. 22-28). 
der Schulbildung und auch des literarischen Lebens. Der Aufschwung der Rhetorik stand im engen Zusammenhang mit dem Pulsieren der Literatur, diese Periode brachte den Individualismus und das Experimentieren, das Spiel mit den Gattungen mit sich. Die Untersuchung von Kazhdan und Epstein wies darauf hin, dass die Erscheinung der Gruppe der Literaten als eine separate professionelle Schicht auch genau auf das 11.-12. Jahrhundert geschätzt werden kann. ${ }^{5}$ Die gelernten Literaten gruppierten sich in literarischen Kreisen, literarischen Salons - oder anders gesagt in theatra ${ }^{6}$ - um die Aristokraten, beziehungsweise um den königlichen Hof, oft um verwitwete Frauen herum. ${ }^{7}$ Diese Salons gaben den Platz und die Gelegenheit dazu, dass die Autoren ihre Werke vorlesen und möglicherweise darüber diskutieren konnten. Also das theatron hat in Byzanz auch de facto eine literarische Rolle und auch eine soziale Funktion - es reicht zu betrachten, wer in den Reihen des Publikums saß: die soziale und geistige Elite aus Konstantinopel, die Lehrer und die Schüler von den Vortragenden. ${ }^{8} \mathrm{Zu}$ dieser Zeit wurden in den literarischen Kreisen in erster Linie rhetorische Übungen (progymnasmata) und rhetorische Stücke vorgetragen, wie die Gedichte von Prodromos. ${ }^{9}$ Da die Oralität in Byzanz sehr wichtig war, war das theatron nach dem „Tod des Theaters“ zugleich auch der Ort der Unterhaltung. Daraus ist gut zu entnehmen, wie eng das literarische Leben im Alltag mit dem gesellschaftlichen Leben verbunden war.

Wenn wir die kleinen Informationen über diese Periode zusammensetzen, ist es klar erkennbar, in welchem engen Zusammenhang die Rhetorik, die byzantinische Literatur, die literarischen Salons und die Patrone miteinander standen. In diese Ordnung fügten sich die Romane sehr gut ein. Die Verfasser waren direkt oder indirekt an den Königshof verknüpft. In den byzantinischen Romanen zeichnet sich die bedeutende Tradition der Oralität und der Aufführung ab, die Autoren bauten in ihre Werke zahlreiche Typen von Progymnasmata ein.

5 Kazhdan \& Epstein (1985: p. 131), über den gesellschaftlichen Zustand der Literaten in Byzanz: ebenda $130-133$.

6 In den literarischen Quellen kommt der Begriff theatron in vielen verschiedenen Bedeutungen vor: diese haben miteinander gemein, dass es sich immer um das Vortragen oder Vorlesen verschiedener Texte handelt. Zum Begriff und zu der sozialen Funktion von theatron: Mullett (1984); Magdalino (1993, bes. pp. 336-356); Marciniak (2007).

7 In der Komnenenzeit betätigten sich beispielweise fünf edle Frauen aus dem Hof als Patroninnen: Ex-Basilissa Maria, Anna Dalassena, Eirene Doukaina, Anna Comnena, Sebastokratorissa Eirene. Eine von ihnen, die Frau von Alexios I., Anna Doukaina hatte persönliche Beziehung mit dem Freund und Lehrer von Niketas Eugenianos, mit Theodoros Prodromos, Sebastokratorissa Eirene und mit zwei Romanenautoren: Theodoros Prodromos und Konstantinos Manasses (Mullett, 1984: pp. 177-179). Der einzige bekannte Patron von Eugenianos war Stephanos Komnenos, das Enkelkind von Sebastokrator Isaakios Komnenos (Agapitos, 2006: p. 150).

8 Jeffreys (2014: p. 14).

9 Marciniak (2007: p. 280). 


\section{Performance als Motiv im Roman von Eugenianos}

Das Werk von Eugenianos, Drosilla und Charikles ist der jüngste von den vier Romanen der Komnenenzeit, im Hinsicht aber auf die „Performance-Zeichen“ und die „Performance-Motive“ vielleicht der reichste. Sehen wir zuerst das Letztere. Der Autor setzte die von den antiken Romanen weitervererbten Grundmotive ${ }^{10}$ und das gegebene Thema in eine Umwelt, die sich von den antiken Vorbildern und von den zeitgenössischen Liebesromanen ganz unterscheidet. Er baute eine Umwelt mit bukolischer, anakreontischer Stimmung, mit wenigen typischen bukolischen Figuren (z.B. Kallidemos), aber mit umso mehr Zitaten von Theokrit, ${ }^{11}$ von anakreontischen Gedichten und von der Anthologia Palatina. Die Handlungsführung ist einfach und macht mit längeren oder kürzeren Unterbrechungen Fortschritte. Vom Gesichtspunkt unserer Analyse sind gerade diese Unterbrechungen interessant, und lassen sich folgendermaßen gruppieren: 1. die in den Faden der Handlung eingefügten Performances, 2. die von der Handlung unabhängigen Szenen und Teile - einerseits, die Exkurse des aktuellen Erzählers, z.B. eine Ekphrasis; andererseits, die verbalen Äußerungen der Figuren, z.B. Monologe, Dialoge, Briefe, kleine Reden, Lieder oder Klagen. ${ }^{12}$ Diese subjektiven Einlagen nehmen eigentlich den größten Teil des Romans ein, indem die Romanfiguren selbst auch zu Erzählern und so zu Vortragenden werden. Aus diesen Teilen erkennen die Leser/Zuhörer die vorhergegangenen Ereignisse, die Geschichte der Bekanntschaft der Liebespaare, mit diesen bringen die Gestalten ihre Gefühle zum Ausdruck. Einige Szenen beschreiben gerade die Atmosphäre und die Tätigkeit der literarischen Salons. Von diesem Gesichtspunkt ist die Episode bemerkenswert, wo Charikles und sein Freund, Kleandros ihre Lebens- und Liebesgeschichte im Gefängnis einander erzählen. Zuerst Kleandros, der die vier Briefe und ein mit Refrain regelmäßig komponiertes, beziehungsweise auf Kithara begleitetes Lied, verfasst über die Liebe, wörtlich zitiert. ${ }^{13}$ Charikles aber kommentiert und wertet manchmal die poetische Leistung des Freundes mit wenigen Worten aus ${ }^{14}$ - wahrscheinlich genauso wie in den theatra. Nach alledem ergreift der Protagonist das Wort und beginnt eine im Thema und in der Thematik zur Rede von Kleandros passende Erzählung. ${ }^{15}$ Charikles, der im Grunde genommen auch selbst an einem kleinen Symposium teilnimmt, erinnert sich an ein anderes, ähnliches Symposium und erzählt darüber. In der Festszene scheint es, dass die Freunde von Charikles ein literarisches Bankett halten,

10 Die Grundmotive der antiken und byzantinischen Romane wurden von Herbert Hunger gruppiert, miteinander verglichen und einander gegenübergestellt (Hunger, 1978: pp. 124-125).

11 Über bukolische Allusionen in Drosilla und Charikles: Burton (2012).

12 P. Roilos im Zusammenhang mit der Oralität in Byzanz: "laments, love and wedding songs, lullabies, narrative songs are attested to have belonged to the repertoire of traditional Byzantine oral poetry" (Roilos, 2012: p. 232). Es ist die Eigenart von Eugenianos, dass er Teile von Theokritidyllen, (oft ganze) anakreontische Gedichte, sowie Epigramme aus der Anthologia Palatina miteinander vermischte und als eine zusammenhängende Einheit in seinen Roman einfügte (Harder, 2012: p. 363).

13 Die Briefe: 2, 169-185; 2, 202-223; 2, 240-277; 2, 284-314; das Lied: 2, 325-385.

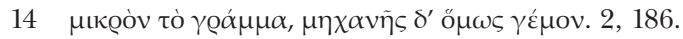

15 Mit kleineren Unterbrechungen: 3, 45-4, 67. 
einer nach dem anderen spricht seine kleine Rede über Eros, Mädchen und Frauen, ${ }^{16}$ ihre Inspirationen aus den vor ihnen spazierenden Frauen geschöpft. ${ }^{17}$ Nach den sechs kleinen Reden werden zwei gut geschriebenen und aus regelmäßigen Strophen bestehenden Lieder mit musikalischer Untermalung vorgetragen. ${ }^{18}$ Diese - zusammen mit den eben erwähnten - sind die ersten Gelegenheiten in den Liebesromanen der Komnenenzeit, dass eine Figur mit Instrument auftritt und singt, obwohl die schriftlichen Quellen davon zeugen, dass die Szene im byzantinischen Hof nicht unbekannt war, ${ }^{19}$ gewiss auch nicht in den theatra. ${ }^{20}$

Wie bereits angedeutet, sind in Drosilla und Charikles noch oft Vorträge zu finden. Es ist daraus wahrnehmbar, dass der Autor immer mit einem Verb oder mit einem Substantiv angibt, nach welcher Gattung, nach welcher Art die Figuren zu reden anfangen. Der performative Aspekt der Einlage ist also in jedem Fall bekannt (z.B. $\alpha_{1}^{\alpha} \sigma \mu \alpha$,

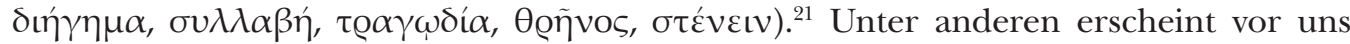
Chrysilla, die Frau des Königs im Parterreich und Verführerin des Charikles als Rhapsodin (5, 183-193), Drosilla als Klageweib (9, 37-107; 9, 235-256), Kleinias, der Sohn von Chrysilla und der Verführer von Drosilla als Sänger (5, 157-219), und nicht zuletzt der Dorfbewohner Kallidemos, ein anderer Verehrer des Mädchens als ein gelernter Rhetor (6, 382-558). ${ }^{22}$ Eugenianos gestaltete diese Episode lebendig und wirkend: der Hauptnarrator wird in den Hintergrund gedrängt, und die Erzähler sprechen immer in direkter Rede.

$\mathrm{Zu}$ den mündlichen Aufführungen kam eine bacchische Tanz-Performance hinzu. Im siebten Buch stellt sich die alte, betrunkene Frau, Baryllis auf den Tisch und tanzt (7, 276-301). Baryllis schafft eine dionysische und karnevalhafte Atmosphäre, die im 12. Jahrhundert in Byzanz sicherlich nicht fremd war. P. Roilos macht darauf aufmerksam, dass zwischen der mittelalterlichen bildenden Kunst, den überlebenden heidnischen

16 Die Szene im Dionysos-Hain wird von Charikles vorgetragen, und auf diese Weise können alle dort rezitierten Lieder und Reden - zumindest indirekt - als von der Hauptperson vorgetragen betrachtet werden. So kann auch von zweier Art Publikum die Rede sein: von einem sogenannten „Intra“, d.h. von dem Erzähler Charikles und seinen Freunden, die all das vorgetragen bzw. „auf direkte Weise“ gehört haben, und einem „Extra“, d.h. von dem, der der Erzählung von Charikles zuhört, also von Kleandros. Ein Beispiel

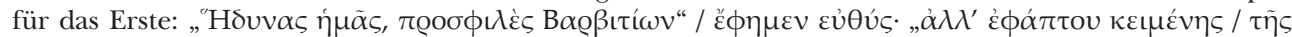

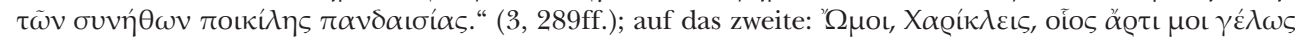

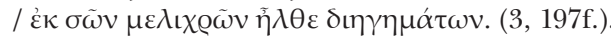

17 Siehe Harder (2012: p. 363).

18 Die Reden: 3, 135-151; 3, 153-161; 3, 163-172; 3, 174-197; 3, 207-241; 3, 243-254; die Lieder: 3, 263-288; $3,297-321$.

19 Agapitos (2006: p. 149).

20 "In such a context, verbal recitation was only part of a total orchestration, in wich architecture, décor, dress, music and choreography also played a part." - P. Magdalino im Zusammenhang mit dem theatron des 12. Jahrhunderts (Magdalino, 1993: pp. 353f.).

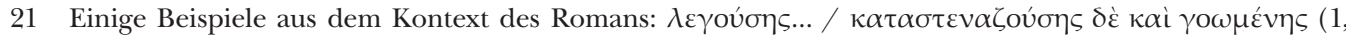

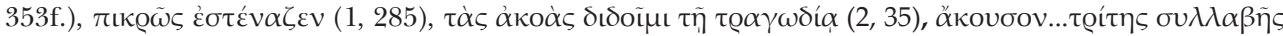

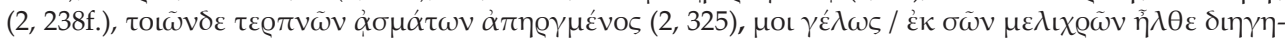
$\mu \alpha ́ \tau \omega \nu(3,197 \mathrm{f}$.$) .$

22 Über die Lamentation als Monolog: Agapitos (2006: p. 348). 
Gewohnheiten und den Dionysosfesten ein Zusammenhang besteht. ${ }^{23}$ Das Publikum sieht in dieser Episode ein neues Genrebild aus dem byzantinischen Alltag. ${ }^{24}$

\section{Der Roman im theatron?}

Bis jetzt ging es darum, wie und wie stark das Motiv der Vortragkunst und das theatron in Drossila und Charikles anwesend sind, und auf welche Art und Weise Eugenianos auf literarische und alltägliche Gewohnheiten reflektiert. Im Weiteren werden diejenigen Merkmale angeführt, die darauf hinweisen, dass dieser Roman oder dessen einzelne Teile mündlich vorgelesen, vorgetragen wurden. Dieses Thema kann von vielen Seiten angenähert werden: von der Rhetorik, von der Struktur des Romans, von der Erzähltheorie, von den Handschriften, von dem Publikum oder auch von den Vortragenden; es ist auch klar, dass diese Annäherungsweisen voneinander nicht immer scharf abgrenzbar sind.

Die Handschriften, die aus dem 13. bis zum 16. Jahrhundert stammen, zeugen davon, dass das literarische Publikum die byzantinischen Romane als rhetorische Werke wahrgenommen hatte. In diesen sind nämlich neben dem Text die Typen der Progymnasmata markiert. In den Romanen kommen die Ethopoiien (als Klagen und Reden), Ekphrasis, Ana- und Kataskeuai, Mythoi und Diegemata besonders häufig vor. ${ }^{25}$ Aller Wahrscheinlichkeit nach verwendeten die Autoren diese rhetorischen Elemente bewusst so häufig, um den Erwartungen und dem Geschmack der zeitgenössischen Leser- und Zuhörerschaft gerecht zu werden, ${ }^{26}$ und das ist ein wichtiger Unterschied im Vergleich zu den antiken Vorbildern.

Der Text von Drosilla und Charikles ist schon auch selbst charakteristisch. Wenn man die narratologischen Merkmale betrachtet, ist es auffallend, dass Eugenianos den Akzent eindeutig auf die Gestalten und deren „Vorträge“ legte. Die Rolle des Hauptnarrators ist neben den anderen Erzählern relativ knapp, sein Auftritt ist hauptsächlich an die Monologe und Dialoge der Romanfiguren gebunden. Wegen der Häufigkeit dieses Phänomens wird der Handlungsablauf selbst in den Hintergrund gedrängt - wie im Fall der Dramen. Der Autor stellte den Haupterzähler ohnehin außerhalb der Geschichte: er ist nämlich ein extra und heterodiegetischer Erzähler, d.h. ein typisch epischer, homerischer Narrator.

P. Agapitos vertritt die Meinung, dass die Struktur der Romane der Komnenenzeit im Zusammenhang mit deren Rezitation in den theatra steht. ${ }^{27}$ Was bedeutet das in

23 Roilos (2005: pp. 292-296). Zur Figur von Baryllis: Roilos (2005: pp. 288-301); Roilos (2012: pp. 232233); Delbó (2015).

24 Es ist eine offene Frage, ob die dramatischen Szenen im theatron vorgetragen wurden und wenn ja, auf welche Art und Weise; und weiters, ob es möglich ist, dass diese als Pantomime dargestellt wurden? Vgl. mit Anm. 20.

25 Harder (2003: p. 360), Conca (1990), ausführliche Analyse über Progymnasmata und ihre Rolle in den byzantinischen Romanen des 12. Jahrhunderts: Roilos (2005: pp. 32-112).

26 Kazhdan \& Epstein (1985: p. 197). Darüber, zu welcher gesellschaftlichen Gruppe das Publikum von Eugenianos wirklich gehörte, sind sehr wenige Informationen vorhanden. P. Agapitos hat den Eindruck, dass die Zuhörerschaft nicht zur höchsten aristokratischen Gesellschaftsschicht gehört, wie es in Verbindung mit Prodromos anzunehmen ist (Agapitos, 2006: p. 150).

27 Agapitos (2006: p. 151). 
Bezug auf das Werk von Eugenianos? Der Roman besteht aus neun Büchern, deren Beginn und Ende oft mit dem Anfang und Ende eines Tages in der Geschichte zusammenklingt (2, 1-4; 7, 1-8; 9, 1f.). ${ }^{28}$ Alle Bücher, wie wir es gesehen haben, bestehen aus vielen Episoden, die Episoden aber gliedern sich in rhetorische Einheiten, in Monologe und Dialoge. Für ein anderes klassisches episches Charakteristikum lässt sich die Anwendung der Formeln nennen, die die Einheiten umrahmen (s.g. „speech-frame formula“). ${ }^{29}$ Diese ein- bzw. zweireihigen Formeln sind vor und hinter einem Monolog angesiedelt, um dessen Selbständigkeit im Text zu betonen, zu markieren, und nicht zuletzt bei der Interpretation zu helfen. In den Anfangsformeln nennt der jeweilige Erzähler, wer spricht und nach welcher literarischen Gattung, oder welchem Stil und welchem Zweck. Die erste Aufgabe der Schlussformeln ist die Überführung von einer Szene zur anderen. Diese fokussieren entweder auf den Vortragenden oder auf das Publikum des Sprechers. Im ersten Fall wiederholt der Narrator, wer spricht, auf welche Art und Weise und was er sagt, im zweiten aber beschreibt er die Wirkung eines Monologs, eines „Vortrags“. 30

Eugenianos widmet seine Arbeit entweder dem Lesen oder der Vorlesung, die Struktur hilft dem Leser oder dem Publikum bei der Interpretation und kann dem Vortragenden auch zu der Rezitation eine Anweisung geben. Dies geschieht nicht nur dadurch, dass jede Szene, jede Texteinheit mit den Formeln sozusagen klar bezeichnet ist, sondern auch dadurch, dass die einzelnen Szenen miteinander mehrmals, durch eine logische Entwicklung verknüpft werden. Es kommt im ersten Buch von Drosilla und Charikles klar zum Ausdruck: Die erste Szene stellt die Belagerung von Barzon dar, wo sich der Fokus regelmäßig zwischen den Ereignissen außerhalb und innerhalb der Stadtmauer wechselt. ${ }^{31}$ Es folgen dann zwei Ekphraseis, ${ }^{32}$ nach denen der Lager des Feindes in den Mittelpunkt gestellt wird - der Lager ist zuerst im Ausland, dann im eigenen Vaterland zu sehen. Das Buch schließt mit zwei Klagen der Protagonisten im gleichen Thema. ${ }^{33}$ Dieses Kapitel beruht auf der parallelen Darstellungsweise, die sowohl in der Makrostruktur als auch in der Mikrostruktur zu beobachten ist.

Was verrät also Drosilla und Charikles? Eine starke Tradition der Vortragkunst, die nicht nur im Text erscheint, sondern mit großer Wahrscheinlichkeit auch in der Rezitation des

28 Agapitos (2006: p. 151).

29 Agapitos (2006: p. 150).

30 Zwei gute Beispiele für die Anfangs- und Schlussformeln: 1) die Klage von Charikles im ersten Buch (1,

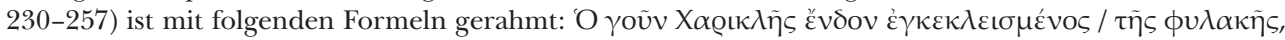

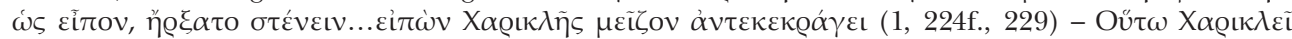

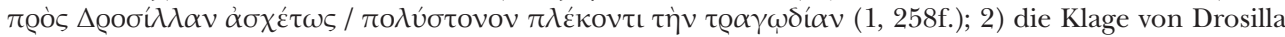

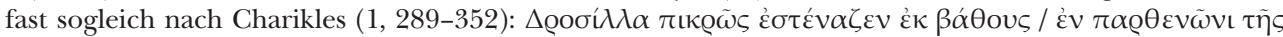

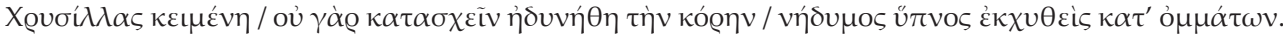

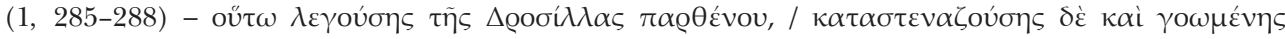
(1,353f.).

31 1-27: außerhalb, 28-41: innerhalb, 47-63: außerhalb, 64ff.: innerhalb.

32 Zuerst über den Altar von Dionysos und seine Umgebung (76-115), dann über Drosilla (120-158).

33 Die Episode im Lager des Feindes: 159-223, die Szene der Klage von Charikles: 224-257, die Klage von Drosilla: 285-355. (Die Klagen zusammen mit den Anfangs- und Schlussformeln bilden eine Einheit.). 
Textes, in den theatra. Ein weiterer Weg der Forschung könnte die vergleichende Untersuchung der Gemeinsamkeiten und Unterschiede der performativen Natur der Romane der Komnenenzeit sein, ausgeführt nach der oben besprochenen Methode. Abschließend sei ein weiteres, interessantes Bindeglied erwähnt.

\section{Kurzer Ausblick}

Im Allgemeinen betrachtet die Fachliteratur das Werk von Eugenianos als eine Nachahmung des Rhodanthe und Dosikles von Prodromos mit einigen Veränderungen, und wird aus diesem Grund bei der Besprechung des Entwicklungsprozesses der Gattung eher ausgegrenzt, obwohl, wie erwähnt, dieser Roman dadurch, dass er der Tradition der bukolischen Dichtung folgt, weitaus der am meisten lyrische Roman unter den zeitgenössischen Werken ist. Genau diese Eigenart und all das, was im Zusammenhang mit dem Performance-Motiv und mit der performativen Natur des Romans eben besprochen worden sind, sind nicht außer Betracht zu lassen. In Livistros und Rhodamne, einem von den acht volkssprachlichen Romanen aus der Paläologenzeit, kann man sehr ähnliche literarische und schriftstellerische Vorgehensweisen feststellen, ${ }^{34}$ z.B.: Kapitel und kleinere Texteinheiten gliedern die Struktur; Briefe, Lieder als Einlage; die häufige Benutzung solcher Wörter, die sich auf das Zuhören oder auf das Sprechen beziehen; die Benutzung von s.g. „speech-frame Formeln“ - diese Formeln geben darüber Auskunft, wer spricht, worüber und nach welcher Gattung. All diese Phänomene deuten einerseits darauf hin, dass der Roman im theatron vorgelesen wurde, andererseits aber darauf, dass zwischen Drosilla und Charikles bzw. Livistros und Rhodamne eine sehr starke Verbindung besteht, die auf eine innere Gattungsentwicklung hinweisen kann. Natürlich ist hier mit der französischen Wirkung auch zu rechnen. Die enge Verbundenheit zwischen den byzantinischen und französischen Romanen, die auf dem Motivschatz und auf der Wiederverwendung der Motive beruht, ist schon bewiesen. Es steht fest, dass die französischen Romane des 12. Jahrhunderts vorgetragen, vorgelesen wurden. Bei der Analyse dieser Werke werden die gleichen Phänomene betont, wie im Fall des Romans von Eugenianos (beispielsweise: wiederkehrende Elemente und Ausdrücke, häufiger Wechsel zwischen den Monologen, Dialogen und den Erzählungen des Hauptnarrators, die Figuren sprechen mit „direct disourse“, kurze Einleitungen vor der Rede einer Figur). ${ }^{35}$ Aus dem Gesichtspunkt der Vortragskunst sind diese noch nicht verglichen worden, obwohl man den Eindruck hat, dass eine solche Untersuchung zu neuen wichtigen Ergebnissen in Hinsicht auf die französisch-byzantinischen literarischen Beziehungen führen würde. Was Drosilla und Charikles betrifft, ist es - meiner Meinung nach - wert, nicht nur die strukturelle Ähnlichkeit zwischen dem Roman von Eugenianus und den chantefable, Aucassin und Nicolette des 12.-13. Jahrhunderts zu erforschen, weil das Letztere in Hinsicht

34 Zur narrativen Form der volkssprachlichen Romane: Agapitos (1991: bes. pp. 64-63). Zu den Performance-Merkmalen: Agapitos (2006: pp. 126-134).

35 Ein zusamenfassender Überblick: Vitz (1999). 
auf die Merkmale der Oralität sowohl mit Drosilla und Charikles, als auch mit Livistros und Rhodamne Ähnlichkeit zeigt. ${ }^{36}$ Es ist aber die Aufgabe der zukünftigen Forschung.

\section{Bibliographie}

Agapitos, P. A. (1991). Narrative Structure in the Byzantine Vernacular Romances. A Textual and Literary Study of Kallimachos, Belthandros and Libistros. München: Institut für Byzantinistik und Neugriechische Philologie der Universität.

Agapitos, P. A. (2006). Writing, Reading and Reciting (in) Byzantine Erotic Fiction. In B. Mondrain (Ed.), Lire et écrire à Byzance (pp. 125-176). Paris: Presses Universitaires de France.

Agapitos, P. A., \& Reinsch, D. R. (Eds.). (2000). Der Roman im Byzanz der Komnenenzeit. Referate des Internationalen Symposiums an der Freien Universität Berlin, 3. bis 6. April 1998 (Meletemata, 8). Frankfurt am Main: Beerenverlag.

Beaton, R. (1996). The Medieval Greek Romance (2nd edition). London: Routledge.

Burton, J. B. (2012). From Theocritean to Longan Bucolic: Eugenianus' Drosilla and Charicles. Greek, Roman, and Byzantine Studies, 52, 684-713.

Conca, F. (Ed.). (1990). Nicetas Eugenianus. De Drosilla et Chariklis amoribus (London Studies in Classical Philology, 24). Amsterdam: J. C. Gieben.

Delbó, K. L. (2015). Hagyomány és újítás a bizánci regényekben: az öreg nő alakja [Tradition und Erneuerung im byzantinischen Roman: die Gestalt der alten Frau]. Antik Tanulmányok, 59, 245-253.

Harder, R. E. (2003). Der byzantinische Roman des 12. Jahrhunderts als Spiegel des zeitgenössischen Literaturbetriebs. In S. Panayotakis, M. Zimmerman, \& W. Keulen (Eds.), The Ancient Novel and Beyond (Mnemosyne Supplementa, 241, pp. 357-369). Leiden - Boston: Brill.

Hunger, H. (1978). Die hochsprachliche profane Literatur der Byzantiner (Vol. II). München: C. H. Beck. Hunger, H. (1980). Antiker und byzantinischer Roman (Sitzungsberichte der Heidelberger Akademie der Wissenschaften, Phil.-hist. Kl., Jahrgang 1980, Abhandlung 3). Heidelberg: Carl Winter.

Jeffreys, E. (2014). Four Byzantine Novels (Translated Texts for Byzantinists, Vol. 1; 2nd edition). Liverpool: Liverpool University Press.

Kazhdan, A. P., \& Epstein, A. W. (1985). Change in Byzantine Culture in the Eleventh and Twelfth Centuries (Transformation of the Classical Heritage, 7). Berkeley - Los Angeles - London: University of California Press.

MacAlister, S. (1996). Dreams and Suicides. The Greek Novel from Antiquity to the Byzantine Empire. London: Routledge.

Magdalino, P. (1993). The Empire of Manuel I Komnenos, 1143-1180. Cambridge - New York: Cambridge University Press.

Marciniak, P. (2007). Byzantine Theatron - A Place of Performance? In M. Grünbart (Ed.), Theatron: Rhetorische Kultur in Spätantike und Mittelalter / Rhetorical Culture in Late Antiquity and the Middle Ages (pp. 277-286). Berlin - New York: de Gruyter.

36 Zur performativen Natur von Aucassin und Nicolette: Vitz (2007). 
Mullett, M. (1984). Aristocracy and Patronage in the Literary Circles of Comnenian Constantinople. In M. Angold (Ed.), The Byzantine Aristocracy: IX to XIII Centuries (BAR International Series, 22, pp. 173-201), Oxford: British Archaeological Reports.

Nilsson, I. (2001). Erotic Pathos, Rhetorical Pleasure. Narrative Technique and Mimesis in Eumathios Makrembolites' Hysmine E゚ Hysminias (Acta Universitatis Upsaliensis. Studia Byzantina Upsaliensia, 7). Uppsala: Uppsala University Library.

Nilsson, I. (2014). Raconter Byzance. La littérature au XIIe siècle (Seminaries Byzantins Series, 3). Paris: Les Belles Lettres.

Roilos, P. (2005). Amphoteroglossia. A Poetics of the Twelfth-Century Medieval Greek Novel (Hellenic Studies, 10). Washington: Center for Hellenic Studies, Trustees for Harvard University.

Roilos, P. (2012). Oral Literature, Ritual, and the Dialectics of Performance. In K. Reichl (Ed.), Medieval Oral Literature (pp. 225-249). Berlin - Boston: de Gruyter.

Vitz, B. E. (1999). Orality and Performance in Early French Romance. Cambridge: D. S. Brewer.

Vitz, B. E. (2007). Variegated Performance of Aucassin et Nicolette. In E. Doss-Quinby, R. L. Krueger, \& E. J. Burns (Eds.), Cultural Performances in Medieval France. Essays in Honor of Nancy Freeman Regalado (pp. 235-245). Cambridge: D. S. Brewer.

Katalin L. Delbó, MA / delbo.katalin@gmail.com

Institute of Ancient and Classical Studies, Greek Department

Eötvös Loránd University, Faculty of Arts

Múzeum krt. 4/F, H-1088 Budapest, Hungary 